\title{
High efficiency laser photothermal excitation of microcantilever vibrations in air and liquids
}

\section{AUTHOR(S):}

Kiracofe, Daniel; Kobayashi, Kei; Labuda, Aleksander; Raman, Arvind; Yamada, Hirofumi

\section{CITATION:}

Kiracofe, Daniel ...[et al]. High efficiency laser photothermal excitation of microcantilever vibrations in air and liquids. REVIEW OF SCIENTIFIC INSTRUMENTS 2011, 82(1): 013702.

\section{ISSUE DATE:}

2011-01

URL:

http://hdl.handle.net/2433/160663

\section{RIGHT:}

Copyright 2011 American Institute of Physics. This article may be downloaded for personal use only. Any other use requires prior permission of the author and the American Institute of Physics. The following article appeared in REVIEW OF SCIENTIFIC INSTRUMENTS 82, 013702 (2011) and may be found at http://link.aip.org/link/?rsi/82/013702 


\section{AIP Scientific Instruments}

\section{High efficiency laser photothermal excitation of microcantilever vibrations in air and liquids}

Daniel Kiracofe, Kei Kobayashi, Aleksander Labuda, Arvind Raman, and Hirofumi Yamada

Citation: Rev. Sci. Instrum. 82, 013702 (2011); doi: 10.1063/1.3518965

View online: http://dx.doi.org/10.1063/1.3518965

View Table of Contents: http://rsi.aip.org/resource/1/RSINAK/v82/i1

Published by the American Institute of Physics.

\section{Related Articles}

Performance improvement of micro-fuel cell by manipulating the charged diffuse layer Appl. Phys. Lett. 101, 113903 (2012)

Surface-tension driven self-assembly of microchips on hydrophobic receptor sites with water using forced wetting Appl. Phys. Lett. 101, 114105 (2012)

Fast acoustic tweezers for the two-dimensional manipulation of individual particles in microfluidic channels Appl. Phys. Lett. 101, 114103 (2012)

Uniform and refreshable liquid electroluminescent device with a back side reservoir APL: Org. Electron. Photonics 5, 208 (2012)

Uniform and refreshable liquid electroluminescent device with a back side reservoir Appl. Phys. Lett. 101, 113302 (2012)

\section{Additional information on Rev. Sci. Instrum.}

Journal Homepage: http://rsi.aip.org

Journal Information: http://rsi.aip.org/about/about_the_journal

Top downloads: http://rsi.aip.org/features/most_downloaded

Information for Authors: http://rsi.aip.org/authors

\section{ADVERTISEMENT}

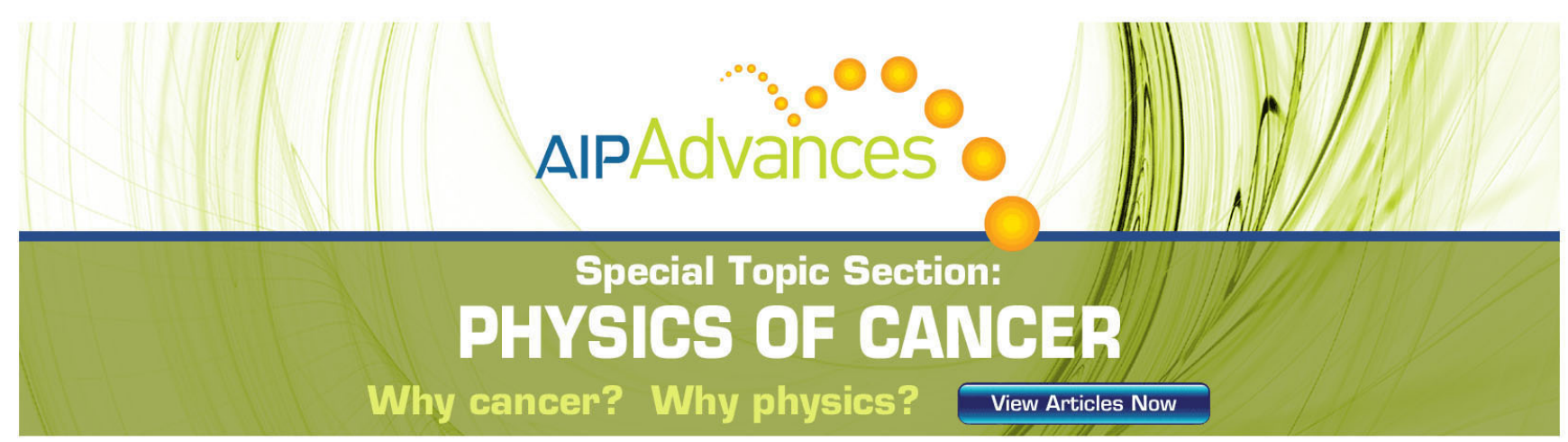




\title{
High efficiency laser photothermal excitation of microcantilever vibrations in air and liquids
}

\author{
Daniel Kiracofe, ${ }^{1}$ Kei Kobayashi, ${ }^{2}$ Aleksander Labuda, ${ }^{3}$ Arvind Raman, ${ }^{1}$ and \\ Hirofumi Yamada ${ }^{4}$ \\ ${ }^{1}$ Department of Mechanical Engineering, Purdue University, West Lafayette, Indiana, 47907 USA \\ ${ }^{2}$ Office of Society-Academia Collaboration for Innovation, Kyoto University, Katsura, Nishikyo, \\ Kyoto 615-8520, Japan \\ ${ }^{3}$ Department of Physics, McGill University, Montreal, Quebec, H3A 2T8 Canada \\ ${ }^{4}$ Department of Electronic Science and Engineering, Kyoto University, Katsura, Nishikyo, \\ Kyoto 615-8510, Japan
}

(Received 23 September 2010; accepted 1 November 2010; published online 3 January 2011)

\begin{abstract}
Photothermal excitation is a promising means of actuating microscale structures. It is gaining increased interest for its capability to excite atomic force microscopy (AFM) microcantilevers with wide frequency bandwidth in liquid environments yielding clean resonance peaks without spurious resonances. These capabilities are particularly relevant for high speed and high resolution, quantitative AFM. However, photothermal efficiency is low, which means a large amount of laser power is required for a given mechanical response. The high laser power may cause local heating effects, or spill over the cantilever and damage sensitive samples. In this work, it is shown that by simply changing from a probe with a rectangular cross-section to one with a trapezoidal cross-section, the photothermal efficiency of an uncoated silicon cantilever can be increased by more than a order of magnitude, and the efficiency of a coated cantilever can be increased by a factor of 2 . This effect is demonstrated experimentally and explained theoretically using thermomechanical analysis. Results are shown for both air and water, and for normal bending and torsional oscillations. () 2011 American Institute of Physics. [doi:10.1063/1.3518965]
\end{abstract}

\section{INTRODUCTION}

Photothermal excitation, the use of an intensitymodulated laser beam to induce mechanical vibration, has been used in micro-electrical mechanical systems (MEMS) actuators $^{1,2}$ and in atomic force microscopy (AFM). ${ }^{3}$ As researchers continue to push the envelope in AFM capability there is growing interest in photothermal excitation due to its advantages over magnetic and acoustic excitation. ${ }^{4,5}$ These advantages include wide frequency bandwidth excitation which is needed for exciting small, high frequency cantilevers used in high speed scanning, ${ }^{6}$ and clean cantilever resonance peaks in liquids free of spurious resonances and phase distortions that enable quantitative force spectroscopy using dynamic AFM. ${ }^{7}$

There are many different mechanisms by which modulated laser light can excite vibrations in microstructures. Silicon (or silicon nitride) AFM cantilevers are typically coated with a thin layer of metal (e.g., gold) to improve reflectivity. In this case, the photothermal excitation mechanism is primarily due to the differing coefficient of thermal expansion and Young's modulus of gold and silicon which creates the wellknown bimorph bending effect. ${ }^{5,8}$ However, it is also possible to use plain, uncoated silicon cantilevers. ${ }^{9}$ Here, thermal gradients through the cantilever thickness creates a bending moment. ${ }^{10}$ For some cases, radiation pressure ${ }^{8}$ and electronic strains ${ }^{12}$ may also contribute to the moment.

One disadvantage of the photothermal method is low efficiency. For coated cantilevers with a blue-violet laser, a typ- ical input power is on the order of milliwatts but output power is on the order of tens of picowatts. For uncoated cantilevers or longer wavelength lasers, efficiency may be one or two orders of magnitude worse. ${ }^{9}$ Because the efficiency is low, the input power must be high. However, this may be undesirable for two reasons. First, high input power leads to local heating of the cantilever and sample, which may cause problems for sensitive experiments. Second, many biological samples are sensitive to light, and high-powered photothermal excitation lasers may damage them. ${ }^{6,13}$ Therefore, it is of interest to determine how to get the most mechanical response for the least amount of laser power.

In this work we will show that the geometry of the cantilever has a large influence on the photothermal response. Specifically, we will show that a trapezoidal cross-section is more than an order of magnitude more efficient than a rectangular cross-section for a plain uncoated cantilever, and a factor of 2 more efficient for a metal coated cantilever. This great difference in photothermal efficiency is due to a higher temperature gradient through the thickness for the trapezoidal cross-section, which is more favorable for bending than the gradients in a rectangular cross-section.

\section{EXPERIMENT: BENDING}

\section{A. Method}

A Shimadzu (Kyoto, Japan) SPM-9600 AFM that has been modified to add a photothermal excitation laser is used for the experiments. A Sanyo $405 \mathrm{~nm}$ blue-violet laser diode 

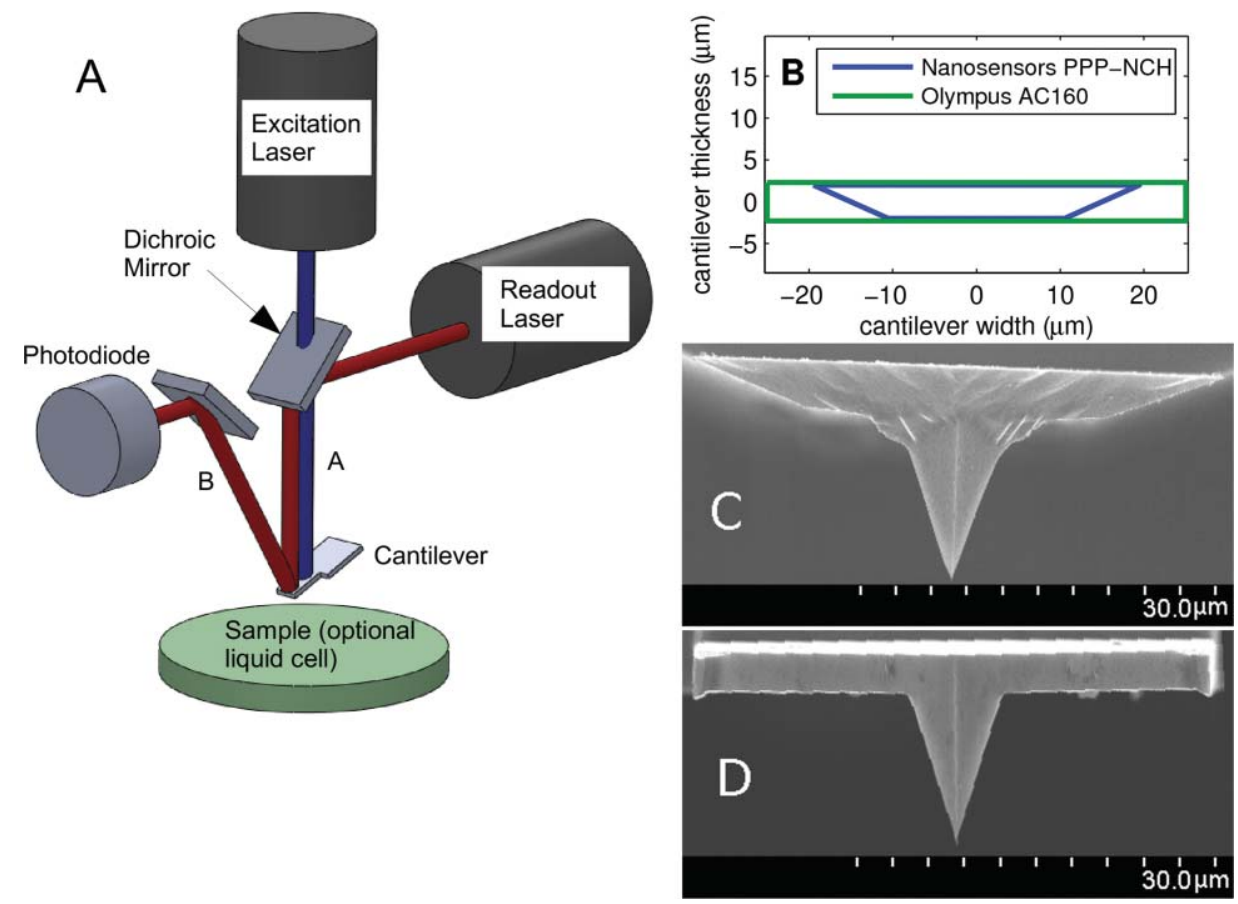

FIG. 1. (Color online) (a) Schematic of experimental setup showing excitation and readout lasers, cantilever, photodiode, and laser intensity measurement locations (marked "A" and "B"). Blue light reflecting off the cantilever is not shown for clarity. A yellow filter at the photodiode removes any reflected blue light. (b) Comparison of the nominal cross-sections for the two different cantilever types. (c) and (d) SEM images of a typical cantilever of each type.

is used for the photothermal excitation (estimated spot diameter $8-18 \mu \mathrm{m}$ ). A schematic of the experimental setup is shown in Fig. 1(a). Two different single-crystal silicon cantilever types are used: Olympus OMCL-AC160-R3 (rectangular cross-section) and Nanosensors PPP-NCH (trapezoidal cross-section). Both gold coated and uncoated versions of each cantilever were tested. Typical parameters of these cantilevers are given in Table I and their cross-sections are compared in Figs. 1(b)-1(d).

The two cantilever types have similar thicknesses, natural frequencies, and quality factors. However, the lengths, widths, and stiffnesses vary between the two types, and even from cantilever to cantilever. ${ }^{14}$ In order to normalize these effects, we report mechanical power $P_{\text {mech }}=\frac{1}{2}\left(k \omega A^{2} / Q\right)$, where $k, \omega, A$, and $Q$ are the first eigenmode stiffness, driving frequency ( $\approx$ first natural frequency), tip oscillation amplitude (peak), and quality factor, respectively. In order to normalize for variations in absorbed, transmitted, and reflected power, a laser power meter was used to measure the excitation laser power incident on the cantilever [location marked A in Fig. 1(a)], reflected from the cantilever (marked B), and transmitted through the cantilever (i.e., incident upon the sample). Across all the cantilevers, the average incident laser power was $7.8 \mathrm{~mW}$, the average transmitted power was $2.2 \mathrm{~mW}$, and average reflected power was $0.9 \mathrm{~mW}$.

The laser power was modulated at each cantilever's first bending natural frequency. Modulation depths were 20\%$40 \%$ in air, and $80 \%$ in water. The responses were normalized by the modulation depth. The data presented are scaled to $80 \%$ modulation depth (i.e., a sine wave with mean value $7.8 \mathrm{~mW}$, minimum value $1.3 \mathrm{~mW}$, and maximum value $14.3 \mathrm{~mW})$.

For each measurement, the photothermal laser spot was adjusted along the length and width until the response was maximized. After that, the laser was moved back and forth across the width of the cantilever.

Sader's method was used to determine the stiffness of each cantilever (using nominal length and width), and then the thermal noise method was used to determine the optical lever sensitivity. ${ }^{15}$

Finally, for observing the laser spot position on the cantilever, the photodiode can be removed and replaced with a digital camera and microscope. This does not allow simultaneous measurement of response and observation of the spot position, but the two could be alternated repeatedly without moving the laser spot or changing the response.

TABLE I. Typical parameters of the cantilevers tested. Dimensions are manufacturer's nominal values in microns (for trapezoidal cantilever, the width is the average of the top and bottom widths). Other parameters are the mean of the tested cantilevers.

\begin{tabular}{|c|c|c|c|c|c|c|c|c|c|c|c|}
\hline \multirow[b]{2}{*}{ Manufacturer } & \multirow[b]{2}{*}{ Type } & \multirow[b]{2}{*}{ Cross-section } & \multirow[b]{2}{*}{ Dimensions } & \multicolumn{3}{|c|}{ Bending, air } & \multicolumn{3}{|c|}{ Torsion, air } & \multicolumn{2}{|c|}{ Bending, water } \\
\hline & & & & $f(\mathrm{kHz})$ & $Q$ & $k(\mathrm{~N} / \mathrm{m})$ & $f(\mathrm{kHz})$ & $Q$ & $k(\mathrm{Nm} / \mathrm{rad})$ & $f(\mathrm{kHz})$ & $Q$ \\
\hline Nanosensors & PPP-NCH & Trapezoidal & $125 \times 30 \times 4.0$ & 236 & 350 & 30.7 & 1910 & 1020 & $8.6 \mathrm{e}-8$ & 140 & 8.6 \\
\hline Olympus & AC160 & Rectangular & $160 \times 50 \times 3.7$ & 288 & 466 & 22.4 & 1450 & 970 & $3.5 \mathrm{e}-7$ & 100 & 7.0 \\
\hline
\end{tabular}




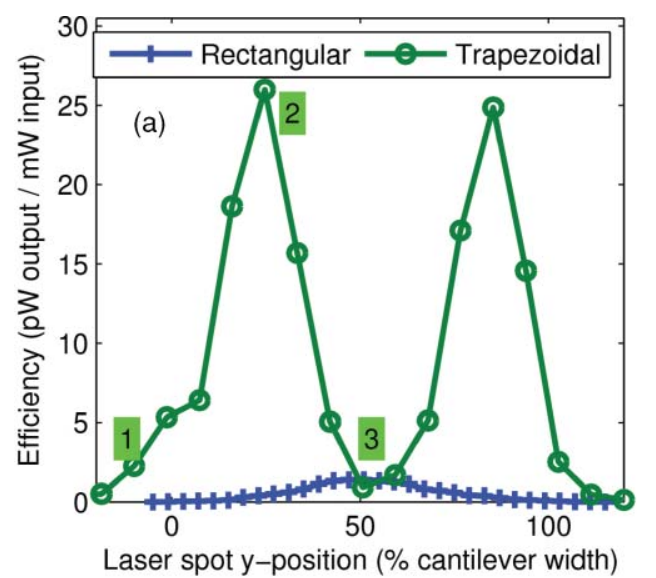

(b)
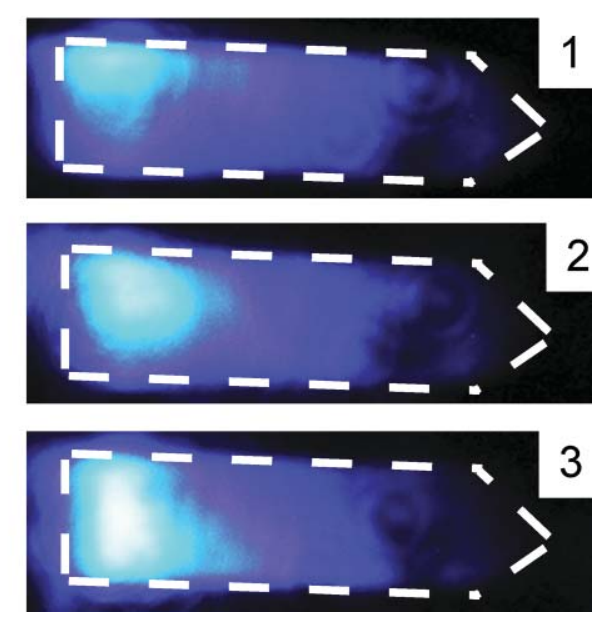

FIG. 2. (Color online) (a) Efficiency of the cantilever (pW mechanical response per $\mathrm{mW}$ absorbed optical power) as a function of laser position along cantilever width for typical uncoated trapezoidal and rectangular cantilevers (the $x$-axis is uncalibrated and serves as a rough guide only). (b) Photographs of the laser spot on the trapezoidal cantilever for three selected positions [corresponding to the marked numbers in (a)]. The white dashed line is an approximate outline of the cantilevers as a guide to the eye.

\section{B. Results}

Figure 2 shows the response of the uncoated trapezoidal and rectangular cantilevers as the excitation laser is swept across the width. Many previous studies ${ }^{4,5,8,9,16}$ of photothermal excitation of cantilevers (one-dimensional models) implicitly assume that this signal should be approximately flat through the middle of the cantilever and fall off toward the edges (where some of the laser light starts to fall off the cantilever), and this result has also been demonstrated in experiments. ${ }^{17}$ This is exactly the response shown by the rectangular cantilevers.

However, the trapezoidal cantilever's response differs from this, showing two distinct peaks near the edges and a valley in the center. Moreover, the response at the sides is nearly 16 times the response at the center. Also, notice that the trapezoidal cantilever has much higher overall efficiency than the rectangular cantilever. The low point at the center of the trapezoidal cantilever is about the same as the peak of the rectangular cantilever. Photographs of the laser spot on the trapezoidal cantilever are also shown in Fig. 2 for the three selected positions as marked, confirming that the position that
TABLE II. Photothermal efficiency ( $\mathrm{pW}$ mechanical power/mW absorbed optical power) for four different cantilever types when driving at the first bending eigenmode in air (laser spot was adjusted to the position that gave maximum response). Error bars are one standard deviation.

\begin{tabular}{ccc}
\hline \hline Cantilever & Uncoated & Coated \\
\hline Rectangular & $1.41 \pm 0.1$ & $64.4 \pm 2.2$ \\
Trapezoidal & $26.5 \pm 5.3$ & $143 \pm 45$ \\
Ratio (Trap/Rect) & 18.8 & 2.2 \\
\hline \hline
\end{tabular}

gives the largest response is when the laser spot is positioned near the edges of the cantilever.

The experiment was replicated with four different cantilevers of each type. The results are given in Table II. There is a statistically significant difference between the photothermal excitation efficiency of the uncoated rectangular and trapezoidal cantilevers - a factor of 19.

Next, the experiment was repeated with four coated rectangular and five coated trapezoidal cantilevers. The results are shown in Table II. As could be expected, the coated cantilever has a higher response than the corresponding uncoated cantilever. This is due to the bimorph bending effect. However, looking only between the two coated cases, the trapezoidal cross-section is still more than a factor of 2 improvement over the rectangular cross-section. We note that the coated trapezoidal cantilevers also have two distinct peaks near the edges and a valley in the center (not shown), but the difference in response between the peak and valley is significantly less (average efficiency of $143 \mathrm{pW}$ out $/ \mathrm{mW}$ in at peak versus 42 at valley - a ratio of 3.4:1 compared to an average of $12: 1$ for uncoated cantilevers).

The remarkable improvement in photothermal excitation efficiency suggests that the thermal gradients in the trapezoidal cantilever are more favorable to bending than in the rectangular cantilever, especially when the laser spot is positioned near the side of the cantilever. Also, the favorable thermal gradients in the trapezoidal cross-section appear to be additive to the bimorph effects. This hypothesis is investigated further in the next section.

\section{ANALYSIS: BENDING}

In the experiment, there were multiple differences between the cantilevers (length, width, manufacturer, etc.) in addition to the different cross-section. In order to verify that the observed phenomena are actually related to the trapezoidal shape, and not some other difference, we analyzed the thermomechanical deflection of the cantilevers. The method of finite elements was used.

\section{A. Modeling}

The method can be separated into two distinct parts. First, the finite element method is used to predict the oscillating temperature amplitude on the microcantilever due to an incident modulated laser beam. Once the temperature amplitude is calculated, a separate mechanical analysis computes the 
oscillating deflection amplitude generated due to the oscillating temperature. In creating the finite element model, we make the following assumptions:

(1) Radiation pressure ${ }^{8}$ is neglected as its effect is small when the excitation laser is focused near the base of the cantilever. $^{17}$

(2) Electronic strain ${ }^{12}$ is neglected as electron diffusion is governed by the same equation as heat diffusion, electronic strain contributions would only affect the results quantitatively and not qualitatively.

(3) Knudsen forces (thermomolecular forces) ${ }^{18}$ are neglected. These are relevant when there is a thermal gradient that has a length scale comparable to the mean free path of the gas molecules. For AFM this typically occurs only under vacuum conditions and the present experiments are in ambient air.

(4) The laser modulation time is on the order of microseconds, so the non-Fourier effect considerations needed for picosecond or femtosecond laser pulses ${ }^{19}$ are not necessary.

(5) The top, bottom, left, and right side boundaries are assumed to be insulated. Based on experimental data presented later (Sec. IV), the results for cantilevers in air and water differ only quantitatively, not qualitatively. That is, the absolute efficiency is different in air versus water, but the ratio of the response for the trapezoidal cross-section versus the rectangular cross-section is the same for both air and water. Therefore any convection heat transfer at the boundary can be ignored if we are only concerned about comparing the cross-sections to each other.

(6) The laser spot is assumed to be far away from any axial boundary (relative to the thermal diffusion length). This simplifies the analysis because the result will be the same no matter where the laser spot is positioned axially. For the conditions of interest, the thermal diffusion length ${ }^{4}$ is short, so this assumption is justified except when the laser spot is positioned within a few micrometers from the end. In the model, the laser is placed half-way along the length.

(7) The laser spot is assumed to be circular (in the experiment, rotating the excitation laser $90^{\circ}$ with respect to the cantilever had no significant effect on the response) with a Gaussian profile such that the electric field is described by $E(r) \propto e^{-(r / w)^{2}}$ and the power (heat generation) is described by $q(r) \propto e^{-2(r / w)^{2}}$, where $r$ is the distance from the spot center and $2 w$ will be referred to as the spot diameter.

Three-dimensional models for rectangular and trapezoidal cantilevers are compared. The analyzed trapezoidal model has dimensions equal to the PPP-NCH cantilever listed in Table I. The model is perfectly uniform along the axial direction (i.e., the cross-section is extruded axially). The analyzed rectangular model has length and thickness identical to the trapezoidal cantilever, and the width equal to the mean width of the trapezoidal cross-section. The analyzed model cantilevers have first natural frequencies that agree with each other within $1.5 \%$ and bending stiffness that agree within $3 \%$.

The MSC NASTRAN finite element solver is used with the transient heat conduction solution routine (the period of the laser modulation is much shorter than the thermal time constant of the system so a steady-state solution cannot be used). A series of runs are conducted for infinitesimal spot sizes (i.e., power applied directly at a node), which are then combined by linear superposition to form the response for a finite spot size. For each laser spot position, a sinusoidal heat generation signal at $300 \mathrm{kHz}$ is applied at the top two nodes. At the wavelength of the violet laser $(405 \mathrm{~nm})$ the $\mathrm{ab}-$ sorption coefficient ${ }^{20}$ is so large that the laser power is reduced to less than $0.5 \%$ by the third node (each element is $235 \mathrm{~nm}$ thick). At the very edge, the trapezoid is only one element thick, so the absorbed power is calculated assuming the laser power that reaches the bottom face is $100 \%$ transmitted (i.e., no internal reflection). ${ }^{21}$ The solution is run for five cycles that are discarded (to let numerical transients settle) and then the last cycle is recorded. Fourier sine and cosine integrals are used to extract an amplitude and phase at each grid point.

The oscillating temperature will cause oscillating thermal expansion [i.e., $\epsilon(x, y, z)=\alpha T(x, y, z)]$. This oscillating thermal strain profile is then used as an input to a forced response mechanical analysis to calculate the oscillating deflection at the free end of the cantilever.

Additional details on the simulations are given in Ref. 18.

\section{B. Result}

\section{Thermal result}

The thermal results for four typical cases are shown in Fig. 3 for the two different geometries. For the first case, the laser spot is centered. The heat oscillation amplitude generated by the laser spot has diffused throughout the thickness and width. There is a small gradient through the thickness underneath the laser spot but for the rest of the cantilever the temperature amplitude is almost uniform through the thickness.

For the second through fourth cases, the laser spot position is moved to the side. For both cross-sections, a gradient is developed along the width. This is because the laser spot is closer to one edge than the other and the edges are insulated. For the rectangular cross-section the gradient through the thickness is essentially unchanged for all four cases. In other words, the top face is hotter (larger temperature oscillation amplitude) in the fourth case than in the first case, but the bottom face is also hotter, so the difference between top and bottom is the same.

However, for the trapezoidal cross-section a strong gradient develops through the thickness. That is, the top face is hotter (larger temperature oscillation amplitude) in the fourth case than in the first case but the bottom face is about the same temperature in both. Essentially, the trapezoidal geometry confines the heat at the top and restricts it from diffusing downward. This temperature gradient increases the bending response. 

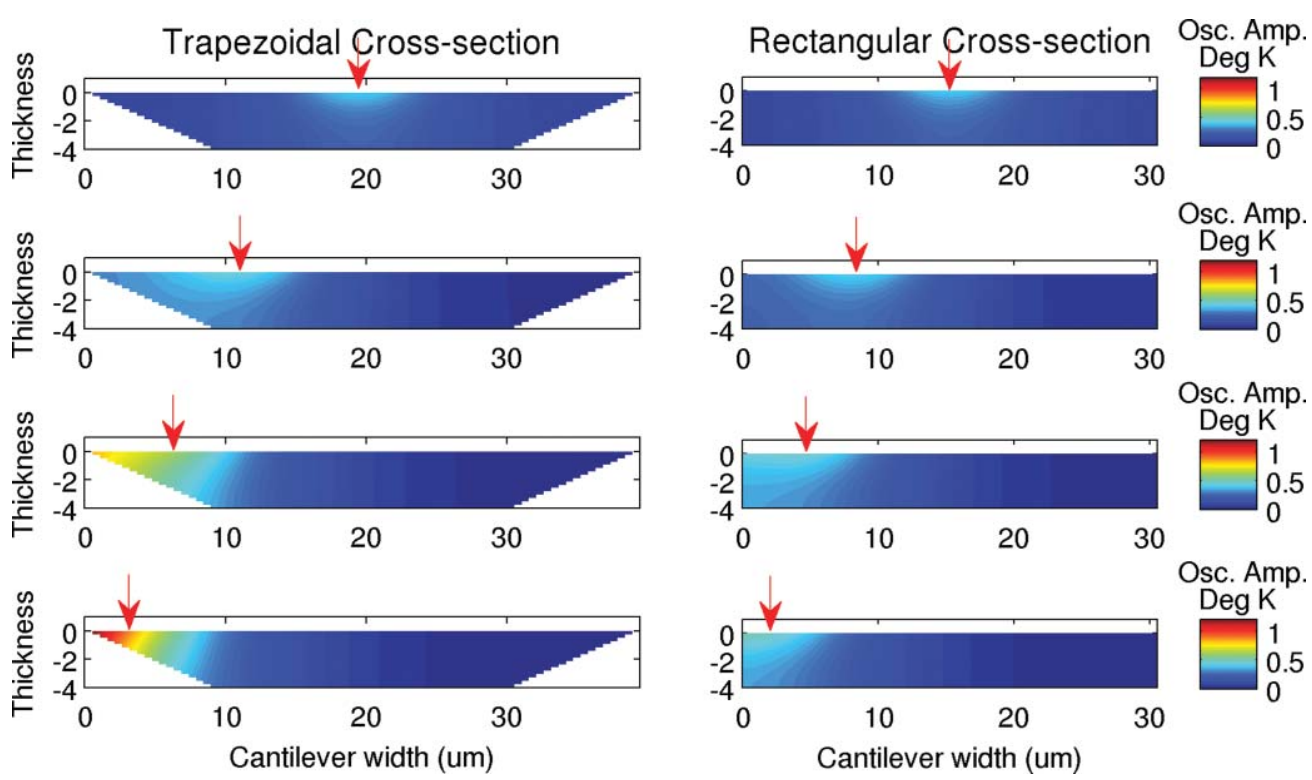

FIG. 3. (Color online) Finite element simulations of temperature oscillation amplitude for four laser spot positions (marked with red arrow), for laser spot diameter $15.3 \mu \mathrm{m}$ and laser power $1 \mathrm{~mW}$. The cross-section at the axial location directly underneath the laser spot is shown. The color bars are degree Kelvin (oscillation amplitude about the mean temperature) and are the same for all plots. For the laser spot in the center, the temperature oscillation amplitude diffuses quickly through both width and thickness. As the laser spot is moved to the side, a gradient along the width develops. The trapezoidal shape forces this gradient to develop into a gradient through the thickness as well (i.e., the hot spot is at the top), which causes bending, but this does not happen for the rectangular cantilever.

\section{Mechanical result}

The resulting vibration amplitudes for different laser spot positions and sizes are shown in Fig. 4. For the trapezoidal cross-section, as the laser spot is moved from the center toward the edge, the oscillating temperature amplitude gradient causes an increase in the vibration amplitude. As the spot is moved farther toward the edge, some laser power begins to spill over the edge due to the finite spot size, which decreases the response. By contrast, the rectangular cantilever shows no such increase at the edge- just a drop off due to the finite spot size. The vibration amplitude of the trapezoidal cantilever is 3-5 times larger than the vibration amplitude of

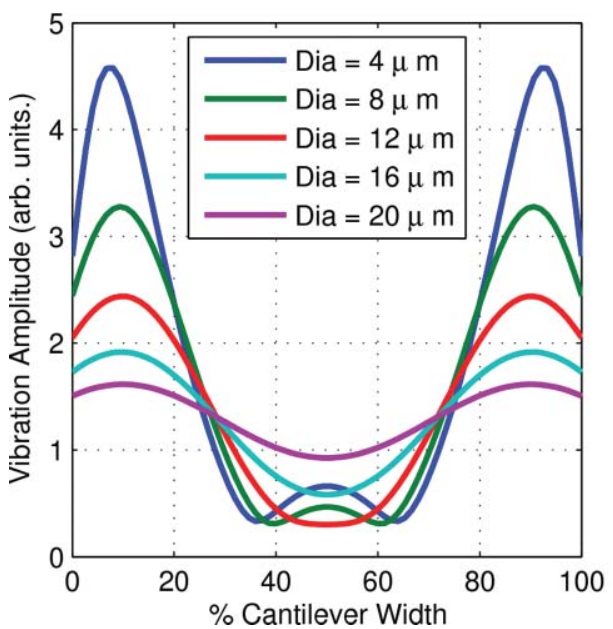

the rectangular cantilever (9-25 times more power) depending on the spot size. These results compare favorably to the experimental data in Fig. 2.

The result suggests that the vibration amplitude is quite sensitive to laser spot size. For smaller spot sizes, three distinct peaks are visible, but for larger spot sizes there are only two. Our typical experimental data in Fig. 2 show two peaks, suggesting that our typical spot size is somewhat large. In Fig. 5, we show the result of attempting to perfect the focus on one cantilever. In the best focus case, the overall vibration amplitude increases and in fact three distinct peaks are visible. It should be noted that this level of focus was difficult to routinely achieve in our instrument.

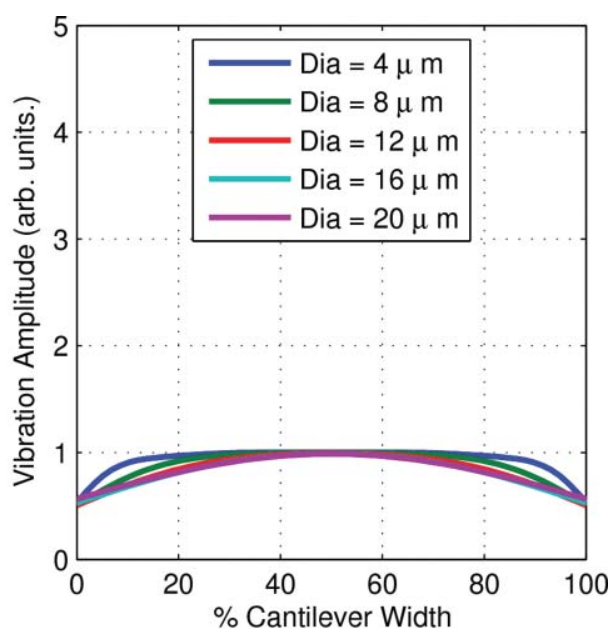

FIG. 4. (Color online) Simulated vibration amplitude vs laser spot position for different laser spot diameters for uncoated cantilevers. Left: Trapezoidal cantilever, Right: Rectangular cantilever. 


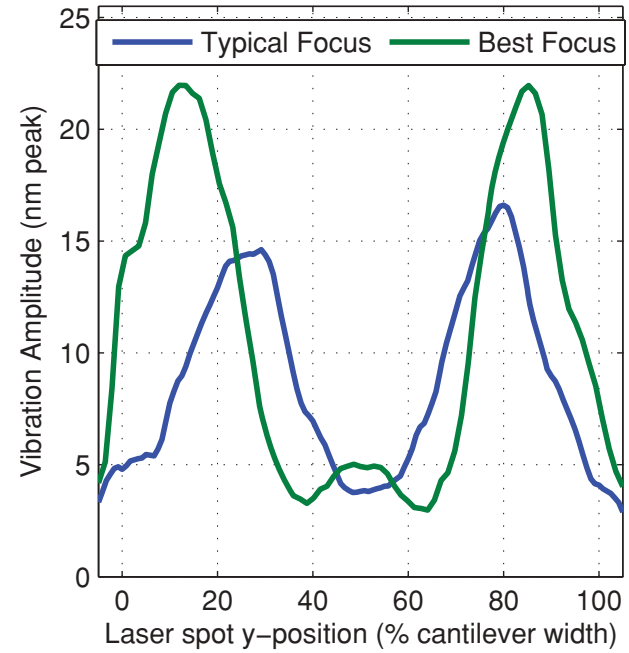

FIG. 5. (Color online) Measured vibration amplitude vs laser spot position for two different focus conditions for a typical uncoated trapezoidal cantilever. For the best focus condition, the vibration amplitude is higher than for the typical focus condition and three distinct peaks are visible instead of two. This is consistent with the simulation in Fig. 4 (the $x$-axis is uncalibrated and serves as a rough guide only).

\section{FURTHER EXPERIMENTS: IN LIQUIDS AND WITH TORSION}

The results presented thus far have clarified the mechanism for the strong increase in photothermal excitation for bending motion in air. It is worth asking if these gains in efficiency also hold in liquid environments, and if the optimum off-center placement of the laser spot for trapezoidal cantilevers can induce some torsional motions in the cantilever?

To answer these questions, we repeated the experiment but immersed the cantilevers in deionized water instead of ambient air. Four cantilevers of each type were used. The natural frequency and quality factor decrease in the liquid environment, as shown in Table I. Also, the laser power meter could not be placed inside the liquid cell, so the values are normalized assuming that the absorbed power is the same for every cantilever and identical to the average value from the experiment in air (reflection at the interface of the water and the top of the liquid cell is assumed to be small). The results are given in Table III. The photothermal efficiency is decreased significantly from the values in air. This decrease in efficiency is likely due to the additional convection heat transfer to the water. However, the important point for the present analysis is the ratio between the response for the rectangular and trapezoidal cross-sections. This ratio is very similar between air and water. For the uncoated cantilever it is 18.1:1 in

TABLE III. Photothermal efficiency (pW mechanical power/mW absorbed optical power) for four different cantilever types when driving at the first bending eigenmode in water.

\begin{tabular}{ccc}
\hline \hline Cantilever & Uncoated & Coated \\
\hline Rectangular & $0.018 \pm 0.003$ & $0.76 \pm 0.02$ \\
Trapezoidal & $0.3 \pm 0.1$ & $1.3 \pm 0.3$ \\
Ratio (Trap/Rect) & 17 & 1.7 \\
\hline \hline
\end{tabular}

TABLE IV. Photothermal efficiency ( $\mathrm{pW}$ mechanical power/mW absorbed optical power) for four different cantilever types when driving at the first torsional eigenmode.

\begin{tabular}{ccc}
\hline \hline Cantilever & Uncoated & Coated \\
\hline Rectangular & $0.3 \pm 0.5$ & $1.5 \pm 0.9$ \\
Trapezoidal & $22 \pm 15$ & $22 \pm 15$ \\
Ratio (Trap/Rect) & 66 & 15 \\
\hline \hline
\end{tabular}

air and 17:1 in water. For the coated cantilever it is $2.2: 1$ in air and 1.7:1 in water. Also, the response as the laser spot is swept across the width (not shown) is similar to the result in air (Fig. 2). Thus we conclude that the trapezoidal cantilever outperforms the rectangular cantilever regardless of media. Because the overall efficiency is lower in liquids, the efficiency gain for the trapezoidal cantilever may be more useful here than in air.

Next, we repeat the experiment but excite the cantilevers at their first torsional eigenmode (in air) and monitor the lateral/torsional deflection of the cantilever. Again, four cantilevers of each type were used. The result is shown in Table IV. The standard deviations are large. However, we can draw some conclusions. First, analogous to the bending results, the trapezoidal cross-section appears to be significantly more efficient than the rectangular cross-section for torsional oscillation. However, in contrast to the bending results, there does not appear to be any statistically significant difference between coated and uncoated cantilevers. Thus, for torsion, an uncoated trapezoidal cantilever can outperform a coated rectangular cantilever. In other words the bimorph effect only weakly excites torsional oscillations but thermal gradients through the thickness strongly excite them.

The laser position which gives the largest response for torsional oscillations is also approximately the laser position which gives the largest response for normal bending oscillations. Thus, researchers may be concerned that torsional oscillations could be unintentionally excited when attempting to excite bending oscillations or vice versa. To test this, we have also monitored the normal bending channel while exciting at the torsional natural frequency, and monitored the torsional channel while exciting the bending natural frequency. Because there may exist some inherent cross-talk in the photodiode signal, we compare these responses to those obtained using piezoexcitation. We have found that for all cases (exciting bending or torsion, in air or in water) the coupling/crosstalk for the photothermal excitation method is the same as or smaller (i.e., better) than for the piezo excitation. Thus, researchers need not be concerned about normal/torsional coupling when using the photothermal method on trapezoidal cantilevers.

\section{DISCUSSION}

The fact that this particular trapezoidal cross-section is superior to a rectangular cross-section suggests that there may exist other geometries which are even more well suited to photothermal excitation. For example, different side-wall angles and different ratios between top and bottom widths may 
improve the response even more. Also, reducing the laser spot size could further increase the response amplitude. Thus, it is conceivable that for a small spot size, a properly designed uncoated trapezoidal cantilever may actually outperform a coated rectangular one in bending.

There are several advantages to using uncoated cantilevers. First, uncoated cantilevers are generally cheaper than coated cantilevers. Second, coated cantilevers suffer from more thermal $\mathrm{drift}^{22}$ and thermal fluctuations at low frequency ${ }^{23}$ than uncoated cantilevers, contributing to noise in experimental measurements. Third, uncoated cantilevers are better than coated cantilevers for exciting higher eigenmodes and very high frequencies. ${ }^{9,24}$

Regardless of whether coated or uncoated cantilevers are used, the present result strengthens the photothermal excitation method against its two main competitors: magnetic and piezoacoustic. The acoustic method introduces many spurious resonance peaks ${ }^{25}$ that make quantitative analysis virtually impossible. The magnetic method becomes difficult at high frequencies due to the impedance of the magnetic coil ${ }^{26}$ and also requires specially coated cantilevers, which in addition to being expensive and corrodible, is not efficient at exciting higher eigenmodes. ${ }^{4}$ The photothermal method has none of these disadvantages. It has a nearly ideal excitation spectrum, does not require any special coating, and works well at higher frequencies and eigenmodes. ${ }^{9}$ It does require the operator to perform an extra step of aligning the laser. One disadvantage though, especially for biological applications, has been the amount of laser power required. The present result significantly reduces this problem. By allowing the same amount of cantilever response for significantly less laser power, the photothermal method has become that much more attractive for biological samples.

\section{CONCLUSION}

To summarize, we have thoroughly investigated the photothermal excitation efficiency of microcantilevers in air and water, for both bending and torsional oscillations. An order of magnitude difference in photothermal efficiency between trapezoidal and rectangular cantilevers has been demonstrated experimentally. The trapezoidal cantilever's response has multiple peaks as the laser is swept across the width, but the rectangular cantilever has only one peak. This result could not have been predicted by existing one-dimensional models. Instead, a three-dimensional thermomechanical model is developed that confirms that the trapezoidal cross-section is responsible for both the efficiency difference and the multiple peaks. This finding demonstrates that large efficiency gains in photothermal excitation efficiency are possible by proper selection of the cantilever geometry thus greatly reducing an obstacle to the increased use of photothermal excitation for applications in atomic force microscopy.

\section{ACKNOWLEDGMENTS}

This research was partially supported by the National Science Foundation through Grant No. 1014660 and CMMI0927648 and by the Japanese Society for Promotion of Science. We would like to thank Dr. Akitoshi Toda, Mr. Masashi Kitazawa, and Mr. Yusuke Nakagawa of Olympus Corporation for providing the Au-coated rectangular cantilevers. D.K. acknowledges the hospitality offered by H. Yamada's lab group during the completion of this research.

${ }^{1}$ T. Lammerink, M. Elwenspoek, and J. Fluitman, IEEE Micro Electro Mechanical Systems, Nara, Japan (IEEE, New York, 1991), pp. 160165 .

${ }^{2}$ K. Thornton, D. Uttamchandani, and B. Culshaw, Electron. Lett. 24, 573 (1988).

${ }^{3}$ N. Umeda, S. Ishizaki, and H. Uwai, J. Vac. Sci. Technol. B 9, 1318 (1991).

${ }^{4}$ D. Ramos, J. Tamayo, J. Mertens, and M. Callega, J. Appl. Phys. 99, 124904 (2006).

${ }^{5}$ G. Ratcliff, D. Erie, and R. Superfine, Appl. Phys. Lett. 72, 1911 (1998).

${ }^{6}$ T. Ando, T. Uchihashi, and T. Fukuma, Prog. Surf. Sci. 83, 337 (2008).

${ }^{7}$ S. Stahl, E. Puchner, and H. Gaub, Rev. Sci. Instrum. 80, 073702 (2009).

${ }^{8}$ O. Marti, A. Ruf, M. Hipp, H. Bielefeldt, J. Colchero, and J. Mlynek, Ultramicroscopy 42, 345 (1992).

${ }^{9}$ S. Nishida, H. Kawakatsu, and Y. Nishimori, J. Vac. Sci. Technol. B 27, 964 (2009).

${ }^{10}$ In the extreme case, such gradients can be strong enough to create permanent plastic deformation (Ref. 11). In the present work we consider only elastic deformations.

${ }^{11}$ X. Zhang and X. Xu, Appl. Phys. Lett. 86, 021114 (2005).

${ }^{12}$ A. Prak and T. Lammerink, J. Appl. Phys. 71, 5242 (1992).

${ }^{13}$ H. Yamashita, N. Kodera, A. Miyagi, T. Uchihashi, D. Yamamoto, and T. Ando, Rev. Sci. Instrum. 78, 083702 (2007).

${ }^{14}$ The rectangular cantilevers were all from two wafers (one for coated and one for uncoated) whereas the trapezoidal cantilevers were sampled from multiple wafers. This leads to more variation in the data for the trapezoidal cantilevers.

${ }^{15}$ M. Higgins, R. Proksch, J. Sader, M. Polcik, S. Mc Endoo, J. Cleveland, and S. Jarvis, Rev. Sci. Instrum. 77, 013701 (2006).

${ }^{16}$ V. Pini, B. Tiribilli, C. Gambi, and M. Vassalli, Phys. Rev. B 81, 54302 (2010).

${ }^{17}$ M. Vassalli, V. Pini, and B. Tiribilli, Appl. Phys. Lett. 97, 143105 (2010).

${ }^{18}$ J. Nabeth, S. Chigullapalli, and A. Alexeenko, 27th International Symposium on Rarefied Gas Dynamics, Pacific Grove, CA, 2010.

${ }^{19} \mathrm{X}$. Wang and X. Xu, J. Therm. Stresses 25, 457 (2002).

${ }^{20}$ D. E. Aspnes, "Properties of Crystalline Silicon," (INSPEC, 1999), Chap. 12.

${ }^{21}$ For longer wavelengths, a significant fraction of light would reach the bottom face over the entire cantilever, which would require a more involved analysis.

${ }^{22}$ M. Radmacher, J. Cleveland, and P. Hansma, Scanning 17, 117 (1995)

${ }^{23}$ P. Paolino and L. Bellon, Nanotechnology 20, 405705 (2009).

${ }^{24}$ See supplementary material at http://dx.doi.org/10.1063/1.3518965 for additional details on simulations and theory.

${ }^{25}$ X. Xu and A. Raman, J. Appl. Phys. 102, 34303 (2007).

${ }^{26}$ Magnetic has been reported to $1 \mathrm{MHz}$ with special design (Ref. 27) whereas photothermal has been reported to $10 \mathrm{MHz}$ (Ref. 9).

${ }^{27}$ M. Kageshima, T. Chikamoto, T. Ogawa, Y. Hirata, T. Inoue, Y. Naitoh, Y. J. Li, and Y. Sugawara, Rev. Sci. Instrum. 80, 23705 (2009). 